\title{
Brown Recluse Spider Bites
}

\author{
Thomas P. Forks, DO, PbD
}

Background: Brown recluse spider bites are a serious medical problem in the southeastern United States. Although most bites are asymptomatic, envenomation can result in a constellation of systemic symptoms referred to as loxoscelism. Patients can also develop necrotic skin ulcers (necrotic arachnidism). These ulcers are often difficult to heal and can require skin grafting or amputation of the bitten appendage.

Methods: A search of the literature was performed using the search words "spider envenomation," "brown recluse spider bites," and "arachnid envenomation."

Results and Conclusions: Most brown recluse spider bites are asymptomatic. All bites should be thoroughly cleansed and tetanus status updated as needed. Patients who develop systemic symptoms require hospitalization. Surgical excision of skin lesions is indicated only for lesions that have stabilized and are no longer enlarging. Steroids are indicated in bites that are associated with severe skin lesions, loxoscelism, and in small children. Dapsone should be used only in adult patients who experience necrotic arachnidism and who have been screened for glucose-6-phosphate dehydrogenase deficiency. Topical nitroglycerin can be of value in decreasing the enlargement of necrotic skin ulcers. (J Am Board Fam Pract 2000;13:415-23.)

Brown recluse spider bites are a relatively common problem in the United States and occur most frequently in the midwest, south-central and southeastern areas of the country. According to the Centers for Discase Control, these bites are not a reportable illness; consequently, the numbers of patients developing systemic or cutaneous manifestations caused by the venom of these spiders can only be guessed. ${ }^{1}$ These bites can result in considerable morbidity and occasionally require the amputation of an involved extremity. Deaths from spider bite, although rare, are usually caused by the induction of renal failure as a consequence of red blood cell hemolysis. Treatment of these bites is controversial and is generally directed at managing the cosmetic injury to the integument. A secondary focus of treatment is the management of the less frequent but potentially more serious systemic effects of the neurotoxic venom, often referred to as loxoscelism. Gangrenous spot, or necrotic spot of Chile (dermonecrosis), as a consequence of the bite

Submitted, revised, 16 February 2000.

From the Department of Family Medicine, The University of Mississippi Medical Center, Jackson. Address reprint requests to Thomas P. Forks, DO, PhD, Department of Family Medicine, The University of Mississippi Medical Center, 2500 North State Street, Jackson, MS 39216-4505. of the venomous South American brown spider (Loxosceles laeta) was initially described by Macchiavello in $1937 .^{2}$ It was not until 1957 that investigation by Atkins et al $^{3}$ implicated venom of the brown recluse spider (Loxosceles reclusa) as the agent of necrotic arachnidism in the United States.

\section{Methods}

A search of the literature was performed using the search words "spider envenomation," "brown recluse spider bites," and "arachnid envenomation."

\section{Brown Recluse Spider}

All spiders are poisonous; however, most have fangs that are too small to penetrate the skin of human beings. There are 23 or 24 genera, including 60 odd species of spiders indigenous to the United States, that have been implicated in causing human injury. Members of at least six of these genera produce bites that can be confused with those of the brown recluse spider. These genera (Atrax, Argiope, Chiracantbium, Lycosa, Pbidippus, Tegenaria) include the wolf, jumping and yellow garden spiders. These spiders are common, well recognized inhabitants of neighborhood backyards and flower gardens. The hobo spider (Tegenaria agrestis) has recently been implicated in cases of necrotic arachnidism in the 
northwest United States. ${ }^{1,4}$ Members of Atrax are not indigenous to the United States. Members of this genus are normally found in eastern Australia and inflict human bites in the United States when imported with Australian produce. Worldwide, there are at least 200 species of spiders that have been implicated in the cutaneous or systemic poisoning of human beings. ${ }^{5}$

Numerous other indigenous North American insects and animals, including kissing bugs, ticks, scorpions, bedbugs, biting flies, fleas, snakes, rats, and mice, produce bites that can be superficially confused with mild cases of loxoscelism. ${ }^{6}$ Other disease states, including herpetic ulcers, burns, trauma, diabetic ulcers, rhus dermatitis, bed sores, pyoderma gangrenosum, Stevens-Johnson syndrome, erythema nodosum, drug reactions, thromboangiitis obliterans, and vascular disease, ${ }^{7-9}$ also produce skin lesions that can be confused with cases of necrotic arachnidism. Consequently, unless the offending animal is presented for identification with the bitten patient, it is prudent to designate all suspected brown recluse spider bites as presumptive brown spider bites, or presumptive necrotic arachnidism until definitive proof is obtained.

Six-eyed spiders (family Loxoscelidae) are represented in the United States by 13 species of spiders. Five of these species ( $L$ arizonica, $L$ reclusa, $L$ rufescens, $L$ deserta, and $L$ laeta) have been implicated in systemic poisoning of bitten patients. These species are all very similar and all five species are appropriately referred to as fiddleback or violin spiders. Although there is some interspecies variation in potency, all Loxosceles species produce a neurotoxic venom that can potentially induce dermonecrosis, varied systemic symptoms, and anaphylaxis in bitten patients. Loxosceles species are found in all the states as a result of translocation on clothing, bagging, and camping gear. Intrastate and interstate relocation of home furnishings and belongings, along with the nests, spiders, and eggs contained therein, is also involved in the movement of these spiders into nonindigenous areas.

The brown recluse spider (brown spider, recluse spider) is the most widely distributed member of the genus in the United States and is the species most often implicated in necrotic arachnidism. $L$ reclusa is indigenous to the states roughly south of the Mason-Dixon line or approximately 42 degrees latitude and east of 100 degrees longitude. These spiders can be found eastward from the western borders of Texas, Oklahoma, and Kansas to the Atlantic coast. Isolated populations of this species can also be found in the more northern states of Nebraska, Iowa, Illinois and Ohio. The species is most concentrated in the central plain states of Arkansas, Kansas, Oklahoma, and Missouri. Specimens have also been identified from California and Hawaii after presumably being transported to those locations with the personal possessions of travelers.

$L$ arizonica is indigenous to Arizona, New Mexico, Nevada, Texas, Utah, southern California, and northwestern Mexico; serious complications secondary to envenomation by this species have also been documented. ${ }^{10} L$ deserta, also known as $L$ unicolor, is relatively uniform in color and its violin is nearly indistinct. This species has been less well studied and is normally found in the deserts of the western states. In the United States, $L$ rufescens is most commonly found in Texas and is referred to by Gertsch ${ }^{11}$ as a cosmopolitan species, ie, a species introduced into the United States from Central America on commercial vehicles.

Envenomations by $L$ rufescens and $L$ laeta are well documented from Central and South America. ${ }^{12} L$ rufipes and $L$ gaucho are also medically important species in Central and South America, respectively. Loxosceles species is represented in Africa by 17 species, and reports of bites on the African continent ( $L$ rufescens, $L$ parrami) have been published in the literature. ${ }^{13}$ Envenomations from Loxosceles species have also been reported from $\mathrm{Eu}$ rope and the Mideast, including Israel, presumably by $L$ rufescens. ${ }^{14}$ Southcott ${ }^{15}$ documented bites from the Australian continent ( $L$ rufescens, $L$ laeta). Envenomations have also been reported by unknown species (probably $L$ rufescens) from China, Russia, and Japan.

\section{Natural History}

Brown recluse spiders are generally shy and inoffensive. These spiders invariably seek shelter in abandoned or infrequently used buildings, in basements, under tables, in dresser drawers, or in stairwells. They can also be found in stored clothing, under wood or rock piles, and within the loosened and flaking bark of trees and logs. They produce small, irregularly formed webs (sheets) that cover and line their burrows or hides. These nocturnal spiders prey on various small invertebrates and insects, including firebrats and related silverfish. ${ }^{16}$ 
Although they have been shown to live up to 3 years in reproduced, artificial environments, their natural life span is probably about 1 year.

Morphologically, female brown recluse spiders are slightly larger than the males and average $9 \mathrm{~mm}$ in length. Older, mature spiders are capable of attaining lengths up to 3 or $4 \mathrm{~cm}$, inclusive of their legs. The body accounts for approximately one fifth of the adult size and is light tannish brown or gray-brown in color. Female brown recluse spiders are generally larger than the males; consequently, they produce a larger amount of venom and their bite is potentially more serious. The distinctive, dark brown or black violin, from which the spider receives its name, is situated on the dorsal aspect of the oval cephalothorax. Individual spiders commonly darken as they age, and the violin can become indistinguishable. The neck of the violin is oriented toward the grayish, oblong, sensillumcovered abdomen of the spider.

In contrast to most spider species, recluse spiders possess six eyes arranged in a semicircle of three diads rather the eight eyes of other species. Adult spiders can live approximately 6 months without eating and can withstand temperature extremes of $8^{\circ}$ to $43^{\circ} \mathrm{C} .^{17}$ Their ability to tolerate such variations in temperature contributes to their survival when accidentally transported to extreme or unusual environments, eg, Finland. Researchers discovered that the entire first floor of a University of Helsinki building was infested with imported $L$ laeta. $^{18}$

\section{Fiddleback Venom}

The collection of adequate volumes of brown recluse venom for biochemical analysis has been difficult and challenging. Researchers have attempted to collect and fractionate venom for study by using electrical stimulation and microdissection of the venom glands. These studies have indicated that the hemolytic component of the venom is heat labile, calcium dependent, and optimally active at a $\mathrm{pH}$ of 7.1. Collectively, research to date has shown that purified brown recluse venom contains a minimum of eight or nine different enzymes and proteins, including alkaline phosphatase, esterase, lipase, protease, hyaluronidase, hemolysins, levarterenol bitartrate, and sphingomyelinase $\mathrm{D}$. Sphingomyelinase D is the most important and most active enzyme in brown recluse spider venom.

\section{Venom Patbophysiology}

Electron microscopic studies of rabbit blood vessels have shown endothelial damage as soon as 3 hours after the manual injection of brown recluse venom. This damage is accompanied by thrombocytopenia, altered prothrombin and partial thromboplastin times, and decreased fibrinogen levels. Shortly thereafter, small capillaries become occluded with thrombi, resulting in further tissue anoxia and destruction. ${ }^{19}$ Anderson ${ }^{20}$ indicated that large quantities of platelets become trapped very early in venom-damaged capillaries. Tissue necrosis is grossly visible at 24 hours after envenomation.

Studies investigating the hemolytic activity of brown recluse venom have produced conflicting results. Material extracted from the cephalothorax of brown recluse spiders has been shown to induce the direct hemolysis of human red blood cells. Cephalothorax extracts contain materials from both the gut and venom glands of spiders. In their 1966 case report, Taylor and Denny ${ }^{22}$ described the clinical course and death of a man who was presumed to have been bitten by a brown recluse spider and who developed hemolysis and renal failure. Smith and Micks ${ }^{23}$ were unable to elicit any hemolytic activity of the venom of $L$ laeta, $L$ reclusa, and $L$ rufescens. Abdominal extracts, however caused the in vitro hemolysis of human red cells. Morgan et $\mathrm{al}^{24}$ reported the in vitro hemolysis of human red blood cells in blood plasma when brown recluse venom was added. Chu et $\mathrm{al}^{25}$ described the case of a 2-year-old boy who was bitten on the chest and who developed a severe case of hemolytic anemia. This patient did not develop a corresponding dermonecrosis. Futrell et $\mathrm{al}^{26}$ proved that a very active component of brown recluse venom attaches to human red cell membranes. They speculated that the resulting lysis of the red blood cells was accomplished through an interaction between this component and complement factors contained in human serum. Conclusive evidence of the lytic activity of brown recluse venom on human red blood cells was described by Forrester et al. ${ }^{27}$ Williams and coworkers ${ }^{28}$ described two cases of severe hemolytic anemia resulting from brown recluse envenomation. One adult female patient suffered a fatal myocardial infarction after debridement of the large necrotic ulcer on her right upper arm.

Morgan and Felton ${ }^{29}$ illustrated human epithelial cell breakdown secondary to brown recluse venom. A generalized breakdown of body fats in 
insects has been shown to occur by Eskafi and Norment ${ }^{30}$ after injection of brown recluse venom. Sphingomyelinase $\mathrm{D}$ is a major component of brown recluse spider venom and has been shown to be cytotoxic to both endothelial cells and red blood cells. ${ }^{27}$ This enzyme has also been shown to induce tissue necrosis in rabbit tissue. ${ }^{31,32}$ Tissue necrosis is directly attributable to the induction of endothelial cell disruption, intravascular hemolysis, platelet aggregation, and thrombi formation by sphingomyelinase $D$. This enzyme is also implicated in the disruption of nerve impulse transmission and in contributing to cutaneous anesthesia. ${ }^{33}$ Tissue necrosis is also partly due to polymorphonuclearleukocyte-induced vasculitis.

The hyaluronidase in the venom contributes to the fluidity of the tissue and resultant spread of venom through the subcutaneous tissue. Gravity might play some role in the spread of the venom and degraded tissue into dependent tissues. Bites in fatty areas seem to fare worse. It has been postulated ${ }^{20}$ that the lipase and other components of the venom act directly on the fatty tissue, releasing small microemboli and nonphysiologic products into the bloodstream.

\section{Clinical Presentation}

When initially bitten by brown recluse spiders, many patients experience a mild burning sensation that might resolve only to reoccur much amplified several hours later. Pruritus, pain, and erythema can develop around the bite site during the first 6 hours after the bite. Hobbs and Harrell ${ }^{34}$ described the formation of an ischemic ring, followed by the development of an irregular erythematous ring surrounding the bite site within the first 24 hours. Some patients, presumably those who have experienced minimal envenomation, are devoid of any discomfort at the time of the bite and are mystified at the subsequent emergence and evolution of skin lesions. Patients are usually bitten when they are dressing themselves by spiders that have previously crawled into their clothing. When bites are immediately painful, the spiders are invariably crushed. All spiders should be brought in by the patient for proper identification even if only partial remains can be collected. Using a hand lens or other suitable magnifying device, the physician might be able to identify the diagnostic six eyes of the spider, thereby making the diagnosis of loxoscelism cer-
Table 1. Systemic and Cutaneous Symptoms of Loxoscelism.

Chills

Fever up to $105^{\circ} \mathrm{F}$

Myalgia and arthralgia

Nausea and emesis

Pain in the bite site

Rash and ulceration

Skin necrosis

Thrombocytopenia

Hemolysis

Disseminated intravascular coagulation

Death

tain. Cacy and Mold ${ }^{35}$ have noted that most brown recluse bites are clinically insignificant.

Systemic symptoms (Table 1) of loxoscelism can include fever up to $105^{\circ} \mathrm{F}^{36}$ chills, nausea, arthritic complaints, and blood abnormalities. ${ }^{37-39}$ Young $^{40}$ reported the interesting case of a 38-year-old man who was bitten by a brown recluse spider and who developed marked thrombocytopenia (platelet count of $13,000 / \mu \mathrm{L}$ ) but no associated hemolysis or disseminated intravascular coagulation. Although the patient had a few petechiae on his lower extremities, his thrombocytopenia subsequently resolved without sequelae. Several authors have also described a generalized erythema or scarlatiniform rash. ${ }^{41-43}$ This rash is generally much more severe in more severely envenomated patients.

Patients with small facial lesions ${ }^{44}$ secondary to brown recluse spider bites have had massive facial edema. Gotto and colleagues ${ }^{45}$ described a case of severe cervical soft-tissue edema and swelling in a 7-year-old boy as a complication of brown recluse spider bite to the neck. $L$ arizonica envenomation has been implicated in the induction of shock in a 13-year-old girl in Arizona. ${ }^{10}$ This patient also developed a dysesthetic, hyperesthetic, sunburn-like rash on her trunk and proximal extremities. Sauer ${ }^{46}$ reported the case of a patient who developed bowel and bladder incontinence, transverse myelitis, and paralysis after a brown recluse spider bite to the right buttock. At discharge, nearly 7 1/2 weeks later, the patient had recovered only partial use of his lower extremities.

Within 24 hours after the bite, the local area around the bite site generally turns a reddish blue color. A blister or bleb then forms, and as necrosis continues, the lesion can evolve into an eschar during the next 2 or 3 days. The eschar will even- 
Table 2. Treatment Regimens for Brown Recluse Spider Bites.

Early and late surgical excision of wound

Wound curettage

Steroids

Dapsone

Hyperbaric oxygen

tually slough off leaving a crater of variable size but generally 1 to $2 \mathrm{~cm}$ wide. Ulcers can become extensive and enlarge to $30 \mathrm{~cm}$ in diameter. Large ulcers might require up to 6 months to heal completely and could require skin grafting. Some patients will develop persistent, granulating ulcers at or near the bite site. ${ }^{36}$ Recurrent ulcers resembling pyoderma gangrenosum can develop years later, even in cases that had originally required treatment with skin grafts.

Grouping the various clinical symptoms of loxoscelism and necrotic arachnidism by severity of systemic symptoms can facilitate treatment. ${ }^{47}$ Such grouping is similar to the grading commonly used when determining the severity of poisonous snake bites. Patients who experience local bite site pruritus and who lack systemic symptoms are in group 1. Group 2 comprises patients whose bites exhibit an area of necrosis approximately $1 \mathrm{~cm}$ or less. The area of necrosis surrounds a central vesicle. Group 3 comprises patients who exhibit definite systemic symptoms and who possess ulcers larger than $1 \mathrm{~cm}$. Group 4 comprises patients who exhibit large ulcers, greater than $4 \mathrm{~cm}$, and who also exhibit severe systemic symptoms. The usefulness of such grading schemes in determining severity of envenomation by fiddleback spiders is questionable because there is little correlation between the severity of skin necrosis and the development of systemic symptoms.

\section{Treatment}

The appropriate treatment of brown recluse spider bites remains controversial, and various treatment regimens (Table 2) have been advocated with varying success. The care of mild, nonprogressive cases of brown spider bite, however, should include thorough cleansing of the wound site, elevation of the bitten extremity, administration of analgesics, and administration of tetanus vaccine, when indicated. Patients should be observed closely to see whether they develop systemic symptoms, at which point immediate hospitalization is indicated.

Surgical correction of venom-induced skin necrosis can add considerable cost to the treatment of violin spider bites. Additionally, it is difficult to determine presumptively which skin lesions will progress to the point where they will require a surgical correction. Hershey and Aulenbacher ${ }^{48}$ advocated treatment with a combination of steroids, antibiotics, and surgery, where indicated. They admitted that none of these three therapies could prevent necrosis of the bite site but suggested that early surgery to remove the involved skin and underlying fat was the preferred therapy. Arnold ${ }^{49}$ promoted surgical excision of all lesions $1 \mathrm{~cm}$ or greater in diameter. Anderson ${ }^{20}$ described lesions that enlarged rapidly. These lesions were reported to cease enlarging suddenly, at which point they developed a well-defined margin.

When surgery is performed, it should be done for lesions that have stabilized, ie, late lesions. The surgeon must be reasonably confident that no postsurgical enlargement of the lesion will occur. DeLozier et al, ${ }^{50}$ in a retrospective study of 31 patients with hand or upper extremity spider bites, determined that delayed excision of the necrotic lesion was preferable. He found that as many as $20 \%$ of the patients who underwent early excision of their necrotic lesions subsequently developed either a wound breakdown or a functional limitation in the use of the bitten extremity. Hollabaugh and Fernandes ${ }^{44}$ recommended curettage of the necrotic tissue with local anesthesia for treatment of brown spider bites. All 18 of the patients in their study who were treated with curettage had wounds that healed without requiring skin grafting. Gutowicz et $\mathrm{al}^{9}$ advocated wide excision of the necrotic lesion after the area of necrosis became well demarcated, 6 to 8 weeks after the bite. Wright et al, ${ }^{51}$ in a study of 111 patients with suspected brown recluse spider bite, found that most did not need surgery; however, 16 of the 111 patients in their study developed systemic symptoms. Three patients required delayed skin grafting. Two of the 3 patients requiring skin grafting had initially received dapsone therapy for treatment of their spider bite.

Jansen et al, ${ }^{52}$ working with white rabbits, did not find any treatment value of either intramuscular or intralesional administration of methylprednisolone (Depo-Medrol) in the prevention of dermonecrosis. Berger et al ${ }^{19}$ also concluded that large 
doses of steroids had little effect on the progression or development of necrotic arachnidism. Sauer, ${ }^{46}$ however, advocated both intramuscular and intralesional injection of cortisone in the treatment of severe bites.

Anderson ${ }^{20}$ advocated the use of steroids only in severe bites, bites that were associated with loxoscelism, and bites involving small children. For children he specifically recommended using $100 \mathrm{mg}$ of prednisone daily for a minimum of 3 days, pending the results of additional studies to rule out hemolysis and renal failure. In this same publication, Anderson described the development of hemolysis, intravascular hemorrhage, coagulation, hyponatremia, myoglobinuria, hemoglobinuria, and coma in a 5-year-old boy who was bitten on his thigh. Steroid therapy was instituted; however, the patient's decreasing kidney output required peritoneal dialysis. Complete resolution of the sequelae of the spider bite required a full 12 months.

Dapsone has been a cornerstone of the therapy of brown recluse spider bite but has severe side effects. Dapsone has been used to treat brown spider bites because it can inhibit polymorphonuclear leukocyte diapedesis and infiltration into the bite site. Animal studies using dapsone have also had conflicting results. Several authors using venominjected animal models have shown a decrease in the average size of necrotic lesions in the animals treated with dapsone. ${ }^{53,54}$ Rees et $\mathrm{al}^{36}$ in a comparative study found that the combination of dapsone and delayed surgical excision was preferable to immediate surgical resection for the treatment of spider bite. Beilman et al ${ }^{55}$ indicated that dapsone, to be effective, must be administered within 36 hours of the bite. This study showed no benefit to pretreatment of spider bites with dapsone. This study also showed no discernible benefit in giving patients dapsone when they sought treatment more than 36 hours after the bite. Phillips et al, ${ }^{56}$ however, were unable to detect any clinical benefit when using dapsone for the treatment of spider bite.

There are serious side effects associated with the use of dapsone. Almost all patients given dapsone will develop dose-related hemolysis. Agranulocytosis, aplastic anemias, and methemoglobinemia occasionally occur. Methemoglobin is unable to bind oxygen and is produced when more than $1 \%$ of the hemoglobin is oxidized to the ferric $\left(\mathrm{HbFe}^{3+}\right)$ state. The resulting increase in oxygen affinity of oxyhemoglobin shifts the oxyhemoglobin dissociation curve to the left. Methemoglobin levels of $35 \%$ or more can produce shortness of breath, headache, and fatigue. Levels as high as $70 \%$ can result in death of the patient. Patients suffering from methemoglobinemia usually have a bluish tint to their mucus membranes and their nail beds. Peripheral neuropathies, although rare, can also occur as a consequence of dapsone administration. Willie and Morrow ${ }^{57}$ described a patient who developed fever, chills, nausea, headache, myalgia, anorexia, and mild hemoglobinuria after treatment of brown spider bite with dapsone. Dapsone hypersensitivity normally occurs within 6 weeks of starting the drug and resolves within 2 weeks of discontinuing therapy. This syndrome does not appear to be dose related.

Other commonly seen side effects of dapsone therapy include sore throat, pallor, purpura, cholestatic jaundice, and hyperbilirubinemia. Many of these symptoms can also be a result of brown spider envenomation, thereby confusing the clinical picture. Because of these serious side effects, dapsone should be reserved for adult patients who have rapidly progressing, necrotic lesions. It should not be administered to children. Adults who are selected to receive dapsone must first be screened for glucose-6-phosphate dehydrogenase deficiency. Dapsone should be given at dosages of 50 to 100 mg daily. ${ }^{53,58}$

The effect of hyperbaric oxygen in decreasing the size of necrotic skin lesions resulting from brown recluse envenomation has also been studied. One study ${ }^{59}$ using white rabbits showed an earlier reepithelization of the necrotic ulcers when these rabbits were treated with hyperbaric oxygen twice daily. Treatments were initiated at 72 hours after injection of venom. Maynor et $\mathrm{al}^{60}$ speculated that hyperbaric oxygen inactivated sphingomyelinase $\mathrm{D}$ by the disruption of sulfhydryl groups. Hyperbaric oxygen has also been postulated to decrease wound damage secondary to brown recluse envenomation in at least two additional ways. ${ }^{56}$ It has been speculated that wound damage is decreased in part because of the pulmonary sequestration of neutrophils. Hyperbaric oxygen therapy also increases the production of collagen by fibroblasts, thereby facilitating wound healing. Beilman et $\mathrm{al}^{55}$ found that guinea pig models which had hyperbaric oxygen therapy as pretreatment had significantly smaller areas of necrosis when compared with control an- 
imals or animals given dapsone as pretreatment. Animals that had hyperbaric oxygen therapy after the injection of recluse venom did not show a corresponding decrease in skin necrosis. Phillips et $\mathrm{al},{ }^{56}$ however, using white rabbits, were unable to show any decrease in lesion size when compared with controls. These animals were injected with 20 $\mu \mathrm{g}$ of $L$ laeta venom and underwent hyperbaric oxygen therapy 4 hours after the injection. In a similar manner, Phillips and colleagues were also unable to show any benefit from treatment with cyproheptadine or dapsone.

Electric shock therapy has also received attention as a possible mechanism of inactivating the enzymes contained in brown recluse venom. ${ }^{54}$ As with snake envenomation, using electric shock to treat spider envenomation is disappointing and cannot be recommended.

Burton $^{61}$ described his 10 years of experience with applying nitroglycerin patches to the bite site area. His experiences indicate that necrosis and ulceration can be aborted if the nitroglycerin patch is applied within 48 hours of the time the patient was bitten. He promoted the use of a $0.1-\mathrm{mg} / \mathrm{h}$ patch and speculated that the nitroglycerin patches prevented necrosis through reversal of blood vessel spasm.

Brown recluse specific antivenin has been shown by Rees et $\mathrm{al}^{31}$ to be most effective for limiting dermonecrosis secondary to brown recluse venom. Similar to antivenin use in the treatment of poisonous snakebite, brown recluse spider antivenin must be administered within the first 24 hours. It is most effective when administered as soon as possible after the bite, unfortunately at a time when the amount of venom injected by the spider is open to speculation. Consequently, it can only be guessed whether the patient received a sufficient quantity of venom to require treatment with antivenin.

\section{Prevention}

Insecticides that are effective in reducing brown recluse spider populations have been shown to be exceedingly toxic to human beings, and their use in human dwellings is ill advised. Reinfestation is a continuous problem, and spiders can hide in areas where insecticides cannot be placed. $^{59}$ Good housekeeping is essential in the prevention of infestation, as the elimination of food sources will decrease infestation. It is also prudent to avoid wearing clothes that have been strewn on the floor during the evening.

\section{References}

1. Necrotic arachnidism-Pacific Northwest, 19881996. MMWR Morb Mortal Wkly Rep 1996;45: 433-6.

2. Macchiavello A. Cutaneous arachnidism or gangrenous spot of Chile. Public Health Trop Med 1947; 22:425-66.

3. Atkins JA, Wingo CW, Sodeman WA. Probable cause of necrotic spider bite in the Midwest. Science 1957;126:73.

4. Blackman JR. Spider bites. J Am Board Fam Pract 1995;8:288-94.

5. Russell FE. A confusion of spiders. Emerg Med 1988;15:6-10.

6. Russel FE, Gertsch WJ. For those who treat spider or suspected spider bites. Toxicon 1983;21:337-9.

7. Pucevich M, McChesney T. Histopathologic analysis of human bites by the brown recluse spider. Arch Dermatol 1983;119:851.

8. Iserson $\mathrm{KV}$. Methemoglobinemia from dapsone therapy for a suspected brown spider bite. J Emerg Med 1985;3:285-8.

9. Gutowicz M, Fritz RA, Sonoga AL. Brown recluse spider bite. A literature review and case report. J Am Podiatr Med Assoc 1989;79:142-6.

10. Bey TA, Walter FG, Lober W, Schmidt J, Spark R, Schlievert PM. Loxosceles arizonica bite associated with shock. Ann Emerg Med 1997;30:701-3.

11. Gertsch WJ. The spider genus Loxosceles in South America. Bull Am Natl Hist 1967;136:121.

12. MacKinnon JE, Witkind J. Arachnidismo necrotico. Ann Fac Med Montevideo 1953;38:75-100.

13. Newlands G, Atkinson P. Review of the southern African spiders of medical importance, with notes on the signs and symptoms of envenomation. S Afr Med J 1988;73:235-9.

14. Efrati P. Bites by Loxosceles spiders in Israel. Toxicon 1969;6:239-41.

15. Southcott RV. Spiders of the genus Loxosceles in Australia. Med J Aust 1976;1:406-8.

16. Luke, Snetsinger. Fiddleback spider-natural predator of firebrats. Sci Agri 1971;18:7. (Au, please add au initials)

17. Hite JM, Gladney WJ, Lancaster JL, Whitcomb WH. Biology of the brown recluse spider. Univ Ark Agr Exp Sta Bull 1966;711:1-26.

18. Huhta V. Loxosceles laeta (Nicolet) (ARANEAE, LOXOSCELINAE), a venomous spider established in a building in Helsinki, Finland, and notes on some other sympathic spiders. Ann Ent Fennici 1972;38: 152.

19. Berger RS, Adelstein EH, Anderson PC. Intravascu- 
lar coagulation-the cause of necrotic arachnidism. J Invest Dermatol 1973;61:142-50.

20. Anderson PC. What's new in loxoscelism-1978: case report. Mo Med 1977;74:549-52, 556.

21. Denny WF, Dillaha CJ, Morgan PN. Hemotoxic effect of Loxosceles reclusus venom: in vivo and in vitro studies. J Lab Clin Med 1964;64:291-8.

22. Taylor EH, Denny WF. Hemolysis, renal failure and death, presumed secondary to bite of brown recluse spider. South Med J 1966;59:1209-11.

23. Smith CW, Micks DW. A comparative study of the venom and other components of three species of Loxosceles. Am J Trop Med Hyg 1968;17:651-6.

24. Morgan BB, Morgan PN, Bowling RE. Lysis of human erythrocytes by venom from the brown recluse spider, Loxosceles reclusa. Toxicon 1978; 16:85-8.

25. Chu JY, Rush CT, O'Connor DM. Hemolytic anemia following brown spider (Loxosceles reclusa) bite. Clin Toxicol 1978;12:531-4.

26. Futrell JM, Morgan BB, Morgan PN. An in vitro model for studying hemolysis associated with venom from the brown recluse spider (Loxosceles reclusa). Toxicon 1979;17:355-62.

27. Forrester LJ, Barrett JT, Campbell BJ. Red blood cell lysis induced by the venom of the brown recluse spider: the role of sphingomyelinase D. Arch Biochem Biophys 1978;187:355-65.

28. Williams ST, Khare VK, Johnston GA, Blackall DP. Severe intravascular hemolysis associated with brown recluse spider envenomation. A report of two cases and review of the literature. Am J Clin Pathol 1995;104:463-7.

29. Morgan PN, Felton WW. Utilization of mammalian cell cultures in spider venom studies. Bacteriol Proc 1965;133:120.

30. Eskafi FM, Norment BR. Physiological action of Loxosceles reclusa (G\&M) venom on insect larvae. Toxicon 1976;14:7-13.

31. Rees RS, Shack RB, Withers EH, Madden J, Franklin J, Lynch JB. Management of the brown recluse spider bite. Plast Reconstr Surg 1981;68:768-73.

32. Rees RS, Nanney LB, Yates RA, King LE Jr. Interaction of brown recluse spider venom on cell membranes: The inciting mechanism? J Invest Dermatol 1984;83: 270-275.

33. Gross AS, Wilson DC, King LE Jr. Persistent segmental cutaneous anesthesia after a brown recluse spider bite. South Med J 1990;83:1321-3.

34. Hobbs GD, Harrell RE Jr. Brown recluse spider bites: a common cause of necrotic arachnidism. Am J Emerg Med 1989;7:309-11.

35. Cacy J. Mold JW. The clinical characteristics of brown recluse spider bites treated by family physicians: an OKPRN study. Oklahoma Physicians Research Network. J Fam Pract 1999;48:536-42.

36. Rees RS, Altenbern DP, Lynch JB, King LE Jr.
Brown recluse spider bites. A comparison of early surgical excision versus dapsone and delayed surgical excision. Ann Surg 1985;202:659-63.

37. Majeski JA, Durst GG Sr. Necrotic arachnidism. South Med J 1976;69:887-91.

38. Weiner RC, Stoffer RP, Chornock FW, et al. Massive intravascular hemolysis. J Kan Med Soc 1960; 61:206-7.

39. James JA, Sellars WA, Austin OA, et al. Reactions following suspected spider bite. Am J Dis Child 1961;102:395-8.

40. Young RA. Thrombocytopenia associated with brown recluse spider bite. J Emerg Med 1994;12: 389.

41. Nance WE. Hemolytic anemia of necrotic arachnidism. Amer J Med 1961;31:801-7.

42. Russell FE, Waldron WG, Madon MB. Bites by the brown spiders Loxosceles unicolor and Loxosceles arizonica in California and Arizona. Toxicon 1969:10917.

43. Atkins JA, Wingo CW, Sodeman WA, Flynn JE. Necrotic arachnidism. Am J Trop Med Hyg 1958;7: 165-84.

44. Hollabaugh RS, Fernandes ET. Management of the brown recluse spider bite. J. Pediatr Surg 1989;24: 126-7.

45. Goto CS, Abramo TJ, Ginsburg CM. Upper airway obstruction caused by brown recluse spider envenomization of the neck. Am J Emerg Med 1996;14: 660-2.

46. Sauer GC. Transverse myelitis and paralysis from a brown recluse spider bite. Mo Med 1975;72:603-4.

47. Auer AI, Hershey FB. Surgery for necrotic bites of the brown spider. Arch Surg 1974;108:612-8.

48. Hershey FB, Aulenbacher CE. Surgical treatment of brown spider bites. Ann Surg 1969;170:300-8.

49. Arnold RE. Brown recluse spider bites: five cases with a review of the literature. JACEP 1976;5:262-4.

50. DeLozier JB, Reaves L, King LE Jr, Rees RS. Brown recluse spider bites of the upper extremity. South Med J 1988;81:181-4.

51. Wright SW, Wrenn KD, Murray L, Seger D. Clinical presentation and outcome of brown recluse spider bite. Ann Emerg Med 1997;30:28-32.

52. Jansen G'T, Morgan PN, McQueen JN, Bennett WE. The brown recluse spider bite: controlled evaluation of treatment using the white rabbit as an animal model. South Med J 1971;64:1194-202.

53. King LE Jr, Rees RS. Dapsone treatment of a brown recluse bite. JAMA 1983;250:648.

54. Barrett SM, Romine-Jenkins M, Fisher DE. Dapsone or electric shock therapy of brown recluse spider envenomation. Ann Emerg Med 1994;24:21-5.

55. Beilman GJ, Winslow CL, Teslow TW. Experimental brown spider bite in the guinea pig. Results of treatment with dapsone or hyperbaric oxygen. J Wilderness Med 1994;5:287-94. 
56. Phillips S, Kohn M, Baker D, et al. Therapy of brown spider envenomation: a controlled trial of hyperbaric oxygen, dapsone, and cyproheptadine. Ann Emerg Med 1995;25:363-8.

57. Wille RC, Morrow JD. Case report: dapsone hypersensitivity syndrome associated with treatment of the bite of a brown recluse spider. Am J Med Sci 1988; 296:270-1.

58. Gendron BP. Loxosceles reclusa envenomation. Am J Emerg Med 1990;8:51-4.
59. Strain GM, Snider TG, Tedford BL, Cohn GH. Hyperbaric oxygen effects on brown recluse spider (Loxosceles reclusa) envenomation in rabbits. Toxicon 1990;29:989-95.

60. Maynor ML, Abt JL, Osborne PD. Brown recluse spider bites: beneficial effects of hyperbaric oxygen. J Hyperbaric Med 1992;7:89-102.

61. Burton KG. Nitroglycerine patches for brown recluse spider bites. Am Fam Physician 1995; $51: 1401$.

\section{Correction}

In the article "Treatment of AIDS and HIVRelated Conditions: 2000" by Ronald H. Goldschmidt and Betty J. Dong (J Am Board Fam Pract 2000;13:274-98), an incorrect Web site was listed for the article in the credit line (p. 274). The article can be found at http:// www.ucsf.edu/warmline/articles/jabfp00.pdf. The authors regret the error.

In a reply to a letter to the editor (Prenatal Testing and Counseling for Down Syndrome. J Am Board Fam Pract 2000;13:2278), Dr. Cate's first name should have been spelled Sara. The publisher regrets the error. 